\title{
Management of intratemporal facial nerve injuries
}

\author{
By U. FISCH, Zürich
}

SKULL fractures involving the temporal bone are, following Bell's palsy, the second most common cause of facial paralysis in adults (17 per cent of the cases) and the most common cause of a facial nerve lesion in children (30.4 per cent of all cases). In spite of this situation and of recent progress in the microsurgical repair of intratemporal facial nerve lesions (Fisch, 1979), confusion still exists as to the proper management of a facial palsy resulting from trauma to the temporal bone. We have treated, at the ENT Department of the University of Zürich, 93 patients with facial paralysis secondary to fractures of the petrous bone. It is the purpose of this paper to present guidelines that have been proved to be of value.

From the clinical point of view, temporal bone fractures are best divided into longitudinal and transverse, according to the preservation or loss of inner ear function. Mixed fractures can be disregarded, because they have to be considered as longitudinal in cases of intact inner ear function, and as transverse in cases of total inner ear loss. Twenty per cent of the longitudinal and 40 per cent of the transverse fractures are accompanied by facial palsy. As long as the post-traumatic palsy remains incomplete, a good prognosis, with total spontaneous return of facial function, is assured. However, in cases with complete clinical paralysis, the patient has to be submitted as early as possible to electrical testing, in order to find out the degree and evolution of degeneration of the motor nerve fibers. For this purpose we advocate electroneuronography (ENoG), because this method alone will provide quantitative information on the number of degenerated facial nerve fibers (Fisch, 1979). Animal experiments have shown that injuries requiring surgical repair will induce the degeneration of more than 90 per cent of motor nerve fibers within 6 days of onset of the palsy. In cases of skull fracture, therefore, surgical exposure of the facial nerve is indicated when ENoG demonstrates more than 90 per cent degeneration within 6 days of the onset of palsy. It is important to realise that the same criterion applies to immediate or to delayed palsy. It is true that a palsy with delayed onset generally carries a better prognosis than an immediate one, but a delayed palsy leading to more than 90 per cent degeneration within 6 days of onset of the palsy (not of the lesion!) may also lead to an irreversible situation.

In our hands, the most reliable topodiagnostic test is Schirmer's test (Fig. 1). However, this test should not be used to identify a 'dry eye', but 
schirmer's test: abnormal values of tearing

UNILATERAL REDUCTION

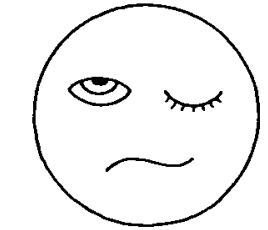

a

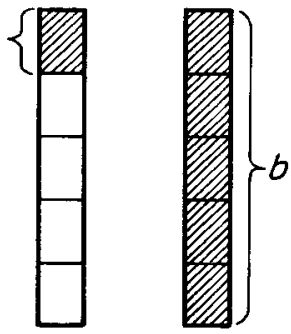

$\frac{b-a}{a+b} \cdot 100>30 \%$
BILATERAL REDUCTION

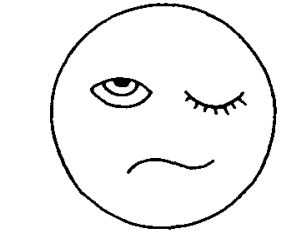

$a$

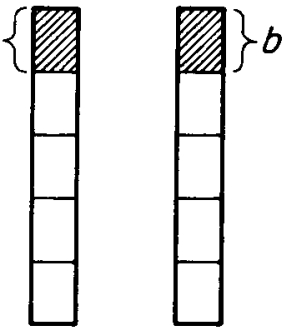

$a+b<25 \mathrm{~mm}$

Fig. 1

Criteria used for the statistical evaluation of Schirmer's test. Note that traumatic rupture of the petrosal nerve leads not only to a unilateral reduction in lacrimation, but also to symmetrically reduced tearing.

\section{Typical longitudinal fracture producing facial paralysis}

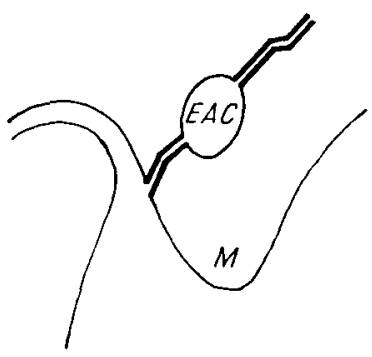

$A_{1}$

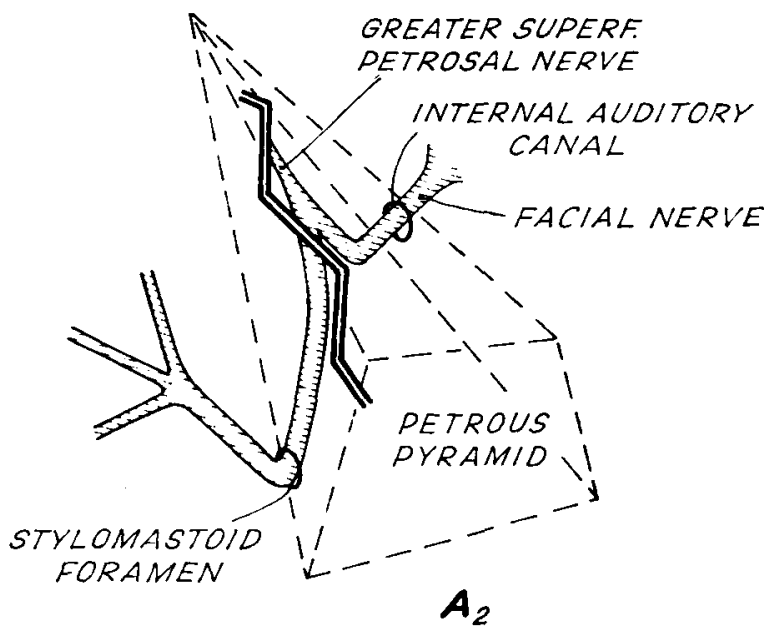

FIG. 2

Schematic representation of longitudinal fracture explaining the high percentage of lesions of the Fallopian canal just distal to the geniculate ganglion. 
it has to be evaluated on a statistical basis. An abnormal degree of lacrimation is present when the secretion is reduced unilaterally by more than 30 per cent of the total lacrimal secretion, or when the total amount of lacrimation (in spite of symmetry) is less than $25 \mathrm{~mm}$. According to this criterion, Schirmer's test was found to be pathological in 93 per cent of
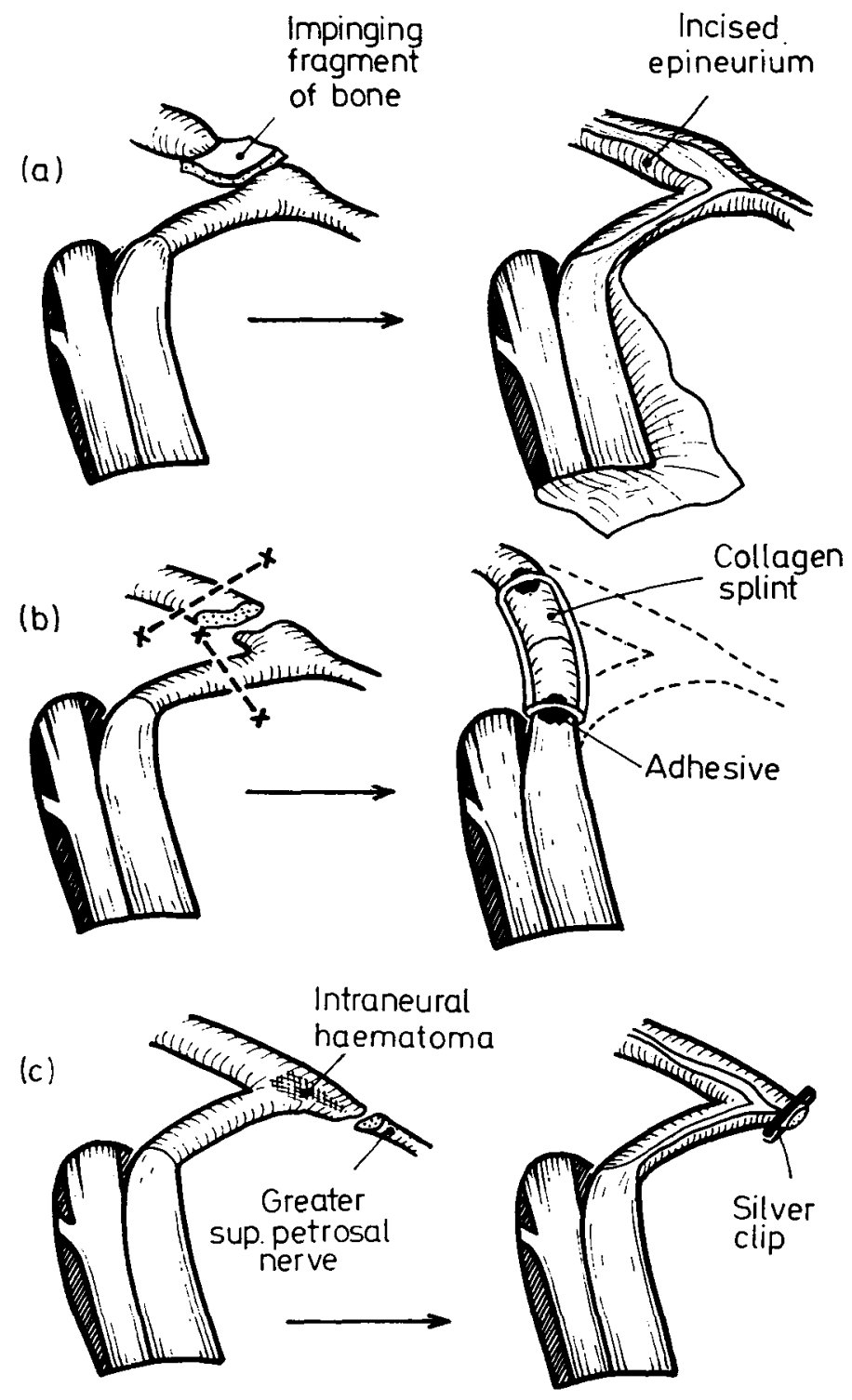

Fig. 3

Most common types of injury found after longitudinal fractures, and their surgical repair. 
patients complying with the above-mentioned indications for surgery. This means that, in order to deal properly with facial nerve injuries due to skull fractures, one has to have a perfect knowledge of the otoneurosurgical procedure.

The high incidence of proximal lesions requiring the middle cranial fossa approach in longitudinal fractures is due to the fact that such fractures usually begin in the temporal squama, extending then across the roof of the middle ear, close to the labyrinthine capsule, and ending in the middle cranial fossa near the carotid canal. Therefore, the Fallopian canal is usually injured (Fig. 2) just distal to the geniculate ganglion. The most common types of lesion that we have observed after longitudinal fractures of the temporal bone are the following:

(a) impression of a bony fragment within the Fallopian canal (20 per cent)

(b) complete section of the facial nerve distal to the genu ( 30 per cent)

(c) intraneural hematoma at the level of the genu ( 50 per cent).

The surgical repair of the above-mentioned lesions consists (Fig. 3) of:

(a) removal of the impinging fragment of bone, with incision of the epineurium of the nerve

(b) re-routing of the Fallopian canal along the superior ampulla, with

\section{Typical transverse fracture producing facial paralysis}

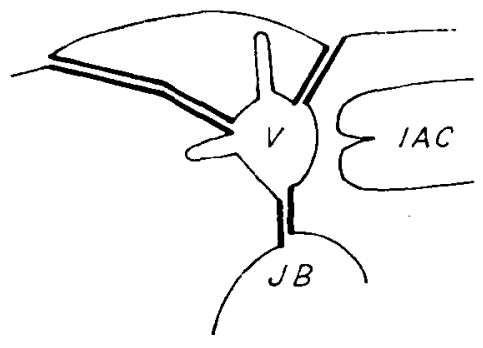

$A_{1}$

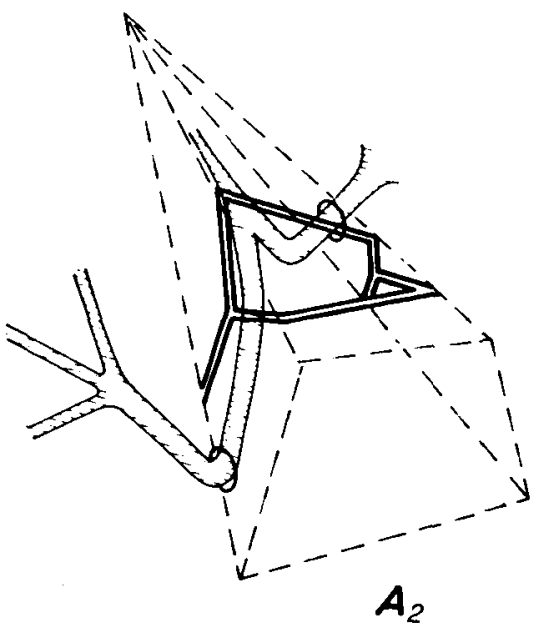

Fig. 4

Schematic representation of a transverse fracture, indicating why the facial nerve is usually injured in two places. 
excision of the genu and end-to-end anastomosis (or nerve grafting)

(c) evacuation of the intraneural hematoma, with incision of the epineurium and clipping of the greater petrosal nerve.

Clipping of the greater petrosal nerve is important, in order to avoid the misdirection of regrowing motor nerve fibers (Fisch, 1979).

Transverse fractures of the pyramid usually extend through the labyrinthine capsule of the temporal bone and, in 70 per cent of cases, they produce a double lesion situated in the proximal labyrinthine segment of the Fallopian canal as well as in its tympanic segment (Fig. 4). The high incidence of a double facial nerve injury requires total exposure of the intratemporal course of the facial nerve, through the translabyrinthine approach, even when a lesion has been seen after exposure of the middle ear. In our hands, what we have called the meato-mastoidal re-routing of the Fallopian canal (Fisch, 1973) is the best method of repair of a facial nerve lesion after transverse fracture of the temporal bone (Fig. 5). Before proceeding to an end-to-end anastomosis or to a nerve grafting procedure, a frozen section of the proximal stump of the facial nerve

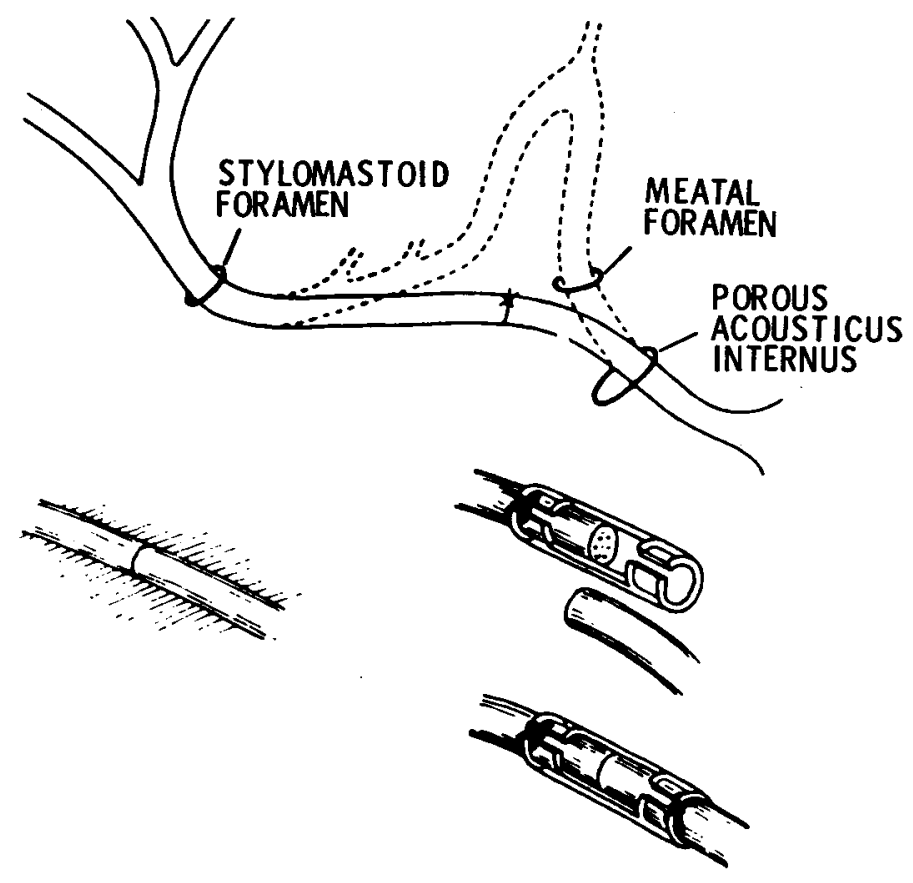

A

B

FIG. 5

The meato-mastoidal re-routing of the facial nerve performed through the translabyrinthine approach is the most successful repair of a facial nerve injury after transverse fracture 
should be studied. If complete fibrosis of the meatal and intracranial segments of the facial nerve has resulted from rupture of the corresponding nutritional vessels, a hypoglossal-facial nerve cross-over is performed.

When is the best time to carry out early surgery? In the presence of an immediate facial paralysis which reaches more than 90 per cent degeneration within 6 days, a delay of 3-4 weeks has given the best results. Conversely, a delayed palsy reaching more than 90 per cent degeneration within 6 days should be operated on immediately, in order to evacuate any intraneural hematoma and to avoid the formation of intraneural fibrosis.

Delayed surgery is indicated if no return or insufficient return of facial function occurs within 6 to 12 months following the injury.

The 2-year follow-up analysis of results has shown that grafting or end-to-end anastomosis of the facial nerve following skull trauma will lead to 60 per cent return of facial movements on average. Evacuation of an intraneural hematoma or removal of an impinging fragment of bone in the Fallopian canal is followed, on average, by an 85 per cent return of facial movements.

\section{REFERENCES}

FisCH, U. (1973) Operations on the facial nerve in its labyrinthine and meatal course. In: Surgery of the Facial Nerve. Ed. A. Miehlke.

Fisch, U. (1979) Facial paralysis. In: Clinical Laryngology. Ed. Maran and Stell, Blackwell Scientific Publications, Oxford-London-Edinburgh, Melbourne. 